\title{
The Generalized Centroid Difference method for lifetime measurements via $\gamma-\gamma$ coincidences using large fast-timing arrays
} \author{
Zs. Podolyák ${ }^{13}$, P.H. Regan ${ }^{13,16}$, O. Stezowski ${ }^{17}$, and A. Vancraeyenest ${ }^{4}$ \\ ${ }^{1}$ IKP der Universität zu Köln, Zülpicher Str. 77, 50937 Köln, Germany \\ ${ }^{2}$ Grupo de Fisica Nuclear, FAMN, Universidad Complutense, CEI Moncloa, 28040 Madrid, Spain \\ ${ }^{3}$ National Centre for Nuclear Research, BP1, ul. Hoza 69, PL-00-681, Warsaw, Poland \\ ${ }^{4}$ LPSC Grenoble, 53, rue des Martyrs, 38026 Grenoble Cedex, France \\ ${ }^{5}$ School of Engineering, University of the West of Scotland, Paisley, PA1 2BE, United Kingdom \\ ${ }^{6}$ Institut Laue-Langevin, CS 20156, 38042 Grenoble Cedex 9, France \\ ${ }^{7}$ GANIL, Bd Henri Becquerel, BP 55027, 14076 Caen Cedex 05, France \\ ${ }^{8}$ INFN, Sezione di Padova, Via Marzolo 8, 35131 Padova, Italy \\ ${ }^{9}$ Faculty of Physics, University of Warsaw, ul. Hoza 69, PL-00-681, Warsaw, Poland \\ ${ }^{10}$ SCEM, University of Brighton, Lewes Road, Brighton, BN2 4GJ, United Kingdom \\ ${ }^{11}$ IKP der Technische Universität zu Darmstadt, Schlossgartenstr. 9, 64289 Darmstadt, Germany \\ ${ }^{12}$ CEA de Saclay, Route Nationale, 91400 Gif-sur-Yvette, France \\ ${ }^{13}$ Faculty of Physics, University of Sofia "St. Kliment Ohridski", 1164 Sofia, Bulgaria \\ ${ }^{14}$ Department of Physics, University of Surrey, Guildford, GU2 7XH, United Kingdom \\ ${ }^{15}$ Horia Hulubei NIPNE, 77125 Bucharest, Romania \\ ${ }^{16}$ NPL, Teddington, Middlesex, TW11 OLW, United Kingdom \\ ${ }^{17}$ IPN de Lyon, 4, Rue Enrico Fermi, 69622 Villeurbanne Cedex, France
}

J.-M. Régis ${ }^{1}$, a , J. Jolie ${ }^{1}$, H. Mach ${ }^{2,3}$, G.S. Simpson ${ }^{4,5}$, A. Blazhev ${ }^{1}$, G. Pascovici ${ }^{1}$, M. Pfeiffer ${ }^{1}$, M. Rudigier ${ }^{1}$, N. SaedSamii $^{1}$, N. Warr ${ }^{1}$, A. Blanc ${ }^{6}$, G. de France ${ }^{7}$, M. Jentschel ${ }^{6}$, U. Köster ${ }^{6}$, P. Mutti ${ }^{6}$, T. Soldner ${ }^{6}$, C.A. Ur ${ }^{8}$, W. Urban ${ }^{6,9}$, A.M. Bruce ${ }^{10}$, F. Drouet ${ }^{4}$, L.M. Fraile ${ }^{2}$, S. Ilieva ${ }^{11}$, W. Korten ${ }^{12}$, T. Kröll ${ }^{11}$, S. Lalkovski ${ }^{13,14}$, N. Mărginean ${ }^{15}$, V. Paziy² ${ }^{2}$,

\begin{abstract}
A novel method for direct electronic "fast-timing" lifetime measurements of nuclear excited states via $\gamma-\gamma$ coincidences using an array equipped with $N$ very fast high-resolution $\mathrm{LaBr}_{3}(\mathrm{Ce})$ scintillator detectors is presented. The generalized centroid difference method provides two independent "start" and "stop" time spectra obtained without any correction by a superposition of the $N(N-1) / 2$ calibrated $\gamma-\gamma$ time difference spectra of the $N$ detector fast-timing system. The two fast-timing array time spectra correspond to a forward and reverse gating of a specific $\gamma-\gamma$ cascade and the centroid difference as the time shift between the centroids of the two time spectra provides a picosecond-sensitive mirror-symmetric observable of the set-up. The energydependent mean prompt response difference between the start and stop events is calibrated and used as a single correction for lifetime determination. These combined fast-timing array mean $\gamma-\gamma$ zero-time responses can be determined for $40 \mathrm{keV}<\mathrm{E}_{\gamma}<1.4 \mathrm{MeV}$ with a precision better than $10 \mathrm{ps}$ using a ${ }^{152} \mathrm{Eu} \gamma$-ray source. The new method is described with examples of $(\mathrm{n}, \gamma)$ and $(\mathrm{n}, \mathrm{f}, \gamma)$ experiments performed at the intense cold-neutron beam facility PF1B of the Institut Laue-Langevin in Grenoble, France, using $16 \mathrm{LaBr}_{3}(\mathrm{Ce})$ detectors within the EXILL\&FATIMA campaign in 2013. The results are discussed with respect to possible systematic errors induced by background contributions.
\end{abstract}

\section{Introduction}

As an essential nuclear observable, the lifetimes of excited states are needed to determine the reduced transition probabilities which are used to test the model dependent intrinsic structure of the nuclear excited states. The electronic fast-timing technique in combination with very fast scintillator detectors is picosecond sensitive [1-3] and therefore is capable of overlapping with complementary techniques such as the Recoil Distance Method [4] and Coulomb exci- tation [5]. For the picosecond regime, the fast-timing technique is based on the determination of centroids of time distributions (first moment of a time spectrum [6]) generated as time difference spectra of consecutive $\gamma-\gamma$ transitions measured using two start and stop $\gamma$-ray detectors. Assuming no background contribution, the experimentally obtained "delayed" time distribution $D(t)$ is a convolution of the Prompt Response Function (PRF) of the setup $P(t)$

a e-mail: regis@ikp.uni-koeln.de 
with an exponential decay as:

$$
D(t)=n \lambda \int_{-\infty}^{t} P\left(t^{\prime}-t_{0}\right) e^{-\lambda\left(t-t^{\prime}\right)} d t^{\prime} \quad \text { with } \quad \lambda=1 / \tau,
$$

where $n$ is the total number of detected $\gamma-\gamma$ events in the time difference spectrum, $\lambda$ the transition (decay) probability and $\tau$ the mean lifetime of the nuclear excited state interconnected by the $\gamma-\gamma$ cascade and $t_{0}$ is the position (centroid) of the PRF $P(t)$. The experimental PRF provides important information on timing characteristics and is obtained for lifetimes which are smaller than 1 ps (systematic errors are expected to be larger). For lifetimes which are larger than the FWHM of the PRF, the mean lifetime is obtained directly using the slope method [1]. Lifetimes which are smaller than the FWHM of the PRF can be determined using the centroid-shift method [6]. For the case of a Gaussian PRF with standard deviation $\sigma \cong \mathrm{FWHM} / 2.355$, the pure statistical time resolving power $\delta t$ of a two detector timing system is given by:

$$
\delta t=\frac{\sigma}{\sqrt{n}} .
$$

The centroid or center of gravity $C^{D}$ is the first moment of the statistical time distribution:

$C^{D}=<t>=\frac{\int_{-\infty}^{\infty} t D(t) d t}{\int_{-\infty}^{\infty} D(t) d t}, \quad \delta C^{D}=\delta t=\sqrt{\left.<t^{2}>-<t\right\rangle^{2}}$,

where $D(t)$ is given by Eq. (1). According to the centroidshift method, the centroid of a delayed time spectrum is displaced by the mean lifetime from the centroid of its convoluted PRF:

$$
\tau=C_{\mathrm{stop}}^{D}-C_{\mathrm{stop}}^{P}
$$

or if the functions of the two detectors are interchanged to obtain the "anti-delayed time spectrum" [1],

$$
\tau=C_{\text {start }}^{P}-C_{\text {start }}^{D},
$$

where $C^{P}$ is the "prompt centroid" of the PRF. The subscript "start" ("stop") indicates that the decay transition with its lifetime information provided the start (stop) timing signal of the two detector timing system.

The centroid-shift method is in principle very simple since the mean lifetime is directly obtained from the centroids of the delayed and the prompt time spectrum. In addition, the centroid-shift method is independent of the shape of the PRF, as demonstrated experimentally in Refs. $[7,8]$. The major problem in $\gamma-\gamma$ fast-timing experiments is that the prompt centroid is dependent on the time response, i.e. the physical zero-time versus energy relation $t_{0}\left(E_{\gamma}\right)$ (the time-walk characteristics) of both detectors of the $\gamma-\gamma$ fast-timing setup, thus $C^{P}=C^{P}\left(E_{\text {start }}, E_{\text {stop }}\right)=$ $t_{0}\left(E_{\text {start }}\right)+t_{0}\left(E_{\text {stop }}\right)$. The calibration of the detector time response is possible, however, the analysis of an $N$ detector timing system becomes complex for $N \gg 2$ as the time response of each single detector has to be calibrated, whereby systematic errors can easily be introduced [9].
The aim of this work is to present the Generalized Centroid Difference (GCD) method, which was developed to provide high-accuracy centroid-shift measurements with an $N$ $\gamma$-ray detector timing system by using a simple approach.

\section{The Generalized Centroid Difference method}

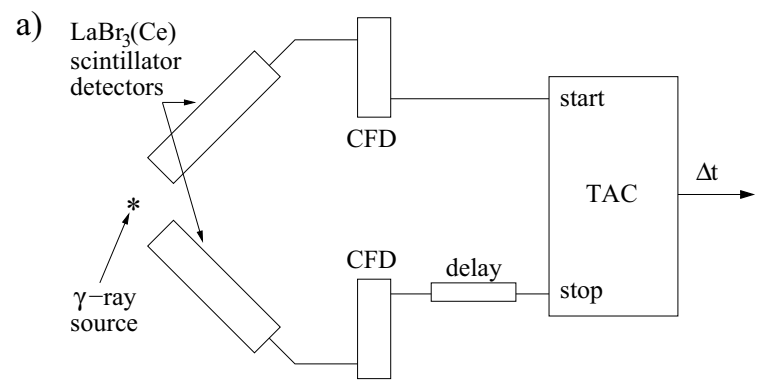

b)

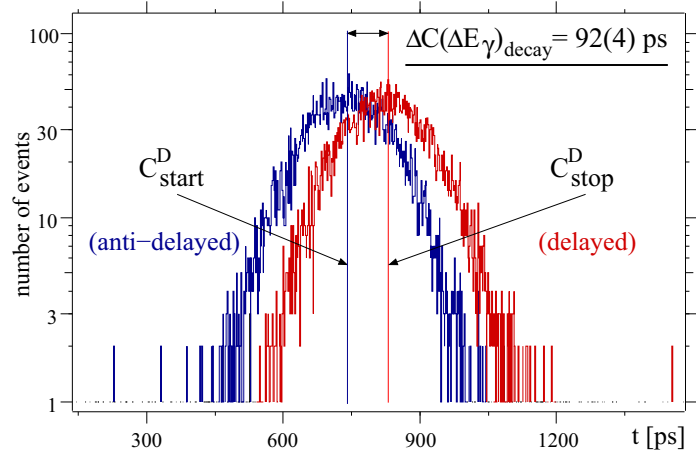

Figure 1. a: The standard electronic $\gamma-\gamma$ fast-timing circuitry using Constant Fraction Discriminators (CFD) for picosecond sensitive time-difference measurements with a Time-to-Amplitude Converter (TAC). b: Two time spectra are obtained dependent on whether the decay transition provided a stop signal (the delayed time spectrum) or a start signal (anti-delayed).

We first consider the standard $\gamma-\gamma$ fast-timing setup consisting of two (start and stop) detectors, as presented in Fig. 1a. As both the start and the stop detectors see the same $\gamma$-ray source, two time distributions are obtained in the off-line analysis by setting a gate (narrow energy window) on the full energy peak of the decay transition $\gamma_{\text {decay }}$ of a nuclear excited state interconnected by a specific $\gamma_{\text {feeder }}-\gamma_{\text {decay }}$ cascade once by using the start detector and once by using the stop detector, while the feeding $\gamma$ ray is detected by the other detector. Assuming no background contributions and according to Eqs. (4) and (5), the time difference between the centroids of the two time spectra presented in Fig. $1 \mathrm{~b}$ corresponds to:

$$
\begin{aligned}
\Delta C\left(\Delta E_{\gamma}\right)_{\text {decay }} & =C^{D}\left(\Delta E_{\gamma}\right)_{\text {stop }}-C^{D}\left(\Delta E_{\gamma}\right)_{\text {start }} \\
& =\operatorname{PRD}\left(\Delta E_{\gamma}\right)_{\text {decay }}+2 \tau,
\end{aligned}
$$

where $\Delta E_{\gamma}=E_{\text {feeder }}-E_{\text {decay }}$ is the energy difference of the two $\gamma$-rays of the cascade and $\operatorname{PRD}\left(\Delta E_{\gamma}\right)_{\text {decay }}=$ $C^{P}\left(\Delta E_{\gamma}\right)_{\text {stop }}-C^{P}\left(\Delta E_{\gamma}\right)_{\text {start }}$ is the Prompt Response Difference which describes the linearly combined $\gamma-\gamma$ zero-time response of the two detector timing system. With respect 
to a start-stop inversion, the centroid difference is mirror symmetric. For the prompt case, this is equivalent to a hypothetical feeder-decay inversion of the cascade [3], thus:

$$
\begin{aligned}
\Delta C\left(\Delta E_{\gamma}\right)_{\text {decay }} & =-\Delta C\left(-\Delta E_{\gamma}\right)_{\text {feeder }}, \\
\operatorname{PRD}\left(\Delta E_{\gamma}\right)_{\text {decay }} & =-\operatorname{PRD}\left(-\Delta E_{\gamma}\right)_{\text {feeder }}
\end{aligned}
$$

and accordingly:

$$
\operatorname{PRD}\left(\Delta E_{\gamma}=0\right)=0 \text { and }\left|\Delta C\left(\Delta E_{\gamma}=0\right)\right|=2 \tau .
$$

Eqs. (6) to (9) represent the Mirror Symmetric Centroid Difference (MSCD) method for a two detector timing system. Due to linear combination of start and stop events, the MSCD method reduces possible systematic errors and cancels the typical systematic error by long term shifts due to drifts in the electronics that can be induced using centroid-shift measurements where the reversed gating is not used. The PRD mirror symmetry, Eq. (8), provides additional data points for a precise calibration of the PRD curve, $\operatorname{PRD}\left(E_{\gamma}\right)$, and makes the determination of the PRD for any energy combination $\Delta E_{\gamma}$ possible using $[3,8]$ :

$$
\operatorname{PRD}\left(E_{\text {feeder }}-E_{\text {decay }}\right)=\operatorname{PRD}\left(E_{\text {feeder }}\right)-\operatorname{PRD}\left(E_{\text {decay }}\right) .
$$

The PRD for the energy combination of a $\gamma-\gamma$ cascade is used as a single correction for the determination of the lifetime according to Eq. (6) and therefore provides the sole uncertainty of the MSCD method, assuming no background contributions to the time spectra.

For the case of a fast-timing array with $N$ almost equal fast-timing detectors and suitable electronics set-up (e.g. the one shown in Ref. [9]), the centroid differences $\Delta C_{i j}$ of all unique detector-detector combinations $i j$ with $j \neq$ $i \in N$ could be measured to provide the mean value as:

$$
\overline{\Delta C}=\frac{2 \sum_{i=1}^{N-1} \sum_{j=i+1}^{N} \Delta C_{i j}}{N(N-1)}=\overline{\mathrm{PRD}}+2 \tau .
$$

Eq. (11) represents the Generalized Centroid Difference (GCD) method and denotes that the time difference between start events (decay transition detected by a start detector) and stop events are statistically distributed around $\overline{\Delta C}$ or $\overline{\mathrm{PRD}}$ independent of the detector-detector combination. Experimentally, the mean centroid difference is equal to the time shift between the two fast-timing-array time spectra generated by a simple superposition of the $N(N-1) / 2$ start (stop) time spectra "TAC ${ }_{i j}$ " [9] (projected time spectra of detector-detector combinations $i j$ ). This identical procedure called the GCD method provides a substantial simplification of fast-timing analyses on $N$ detector timing systems as the tedious determination of $N$ detector time responses is eliminated. The complexity of the data analysis is reduced to that of a two detector timing system. Analogous to the MSCD method, the mean PRD is used as the only correction which reduces a possible systematic error to a minimum by taking advantage of the mirror symmetric representation of the GCD method.

\section{The GCD method for EXILL\&FATIMA}

The GCD method was developed especially for large fasttiming arrays such as the "FATIMA" $\gamma$-ray spectrometer made of $N=165 \% \mathrm{Ce}$ doped $\mathrm{LaBr}_{3}$ detectors that was installed at the Institut Laue-Langevin (ILL) in Grenoble, France. This FATIMA spectrometer was combined with part of the EXILL (EXOGAM at ILL [10]) spectrometer consisting of 8 HPGe EXOGAM Clover detectors. Within the EXILL\&FATIMA campaign, fast electronic timing measurements were performed in prompt $\gamma$ ray spectroscopy experiments using neutron-capture and neutron-induced fission reactions at the highly collimated high-flux cold-neutron beam facility PF1B $[11,12]$ of the ILL. Triple coincidences were used in which a Ge gate selects the cascade of $\gamma$ rays to be measured by the $\mathrm{LaBr}_{3}$ detectors. A detailed description of the EXILL\&FATIMA set-up is given in Ref. [13]. In a preparatory work, the $N(N-1) / 2=120 \mathrm{LaBr}_{3}-\mathrm{LaBr}_{3}$ coincidence spectra of the FATIMA set-up were investigated for artefacts such as inter-detector Compton scattering. Such cross-talk events with false timing information have been detected in 16 adjacent and 8 other $\mathrm{LaBr}_{3}-\mathrm{LaBr}_{3}$ detector combinations, those are excluded from the main data analysis [13].

a)

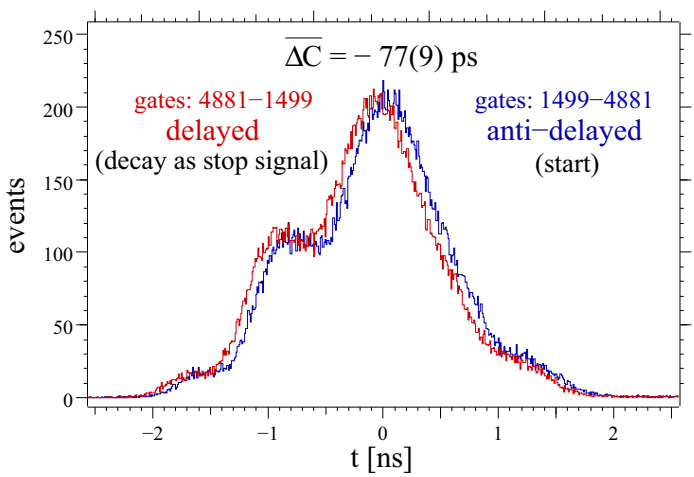

b)

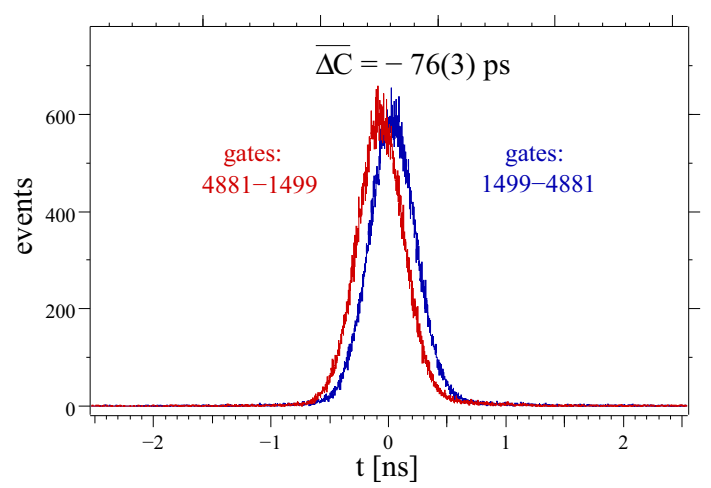

Figure 2. Principle of the GCD method. a: Superposition of 96 remaining time-difference spectra $\mathrm{TAC}_{i j}$ of a prompt $\gamma-\gamma$ cascade in ${ }^{49} \mathrm{Ti}$ with $\tau=16(7)$ fs [14] as obtained from the $16 \mathrm{LaBr}_{3}$ fast-timing array of the EXILL\&FATIMA spectrometer, by distinguishing between the stop events (decay transition detected as a stop signal) and the start events independent of the detectordetector combination $i j$. b: Result of the same data shown in Fig. 2a obtained after the alignment of the $96 \mathrm{TAC}_{i j}$ spectra using constant shift ${ }_{i j}$ values (see the text for more detail) in order to improve the time resolution [FWHM=292(3) ps]. 
Fig. 2 illustrates the principle of the GCD method: The fast-timing data of the 96 unique detector-detector combinations (time spectra $\mathrm{TAC}_{i j}$ ) are superimposed without any correction by distinguishing between the delayed (decay transition detected as stop signal) and the anti-delayed time spectrum of a $\gamma-\gamma$ cascade to measure the centroid difference. In order to improve the precision of the measurement following Eq. (2), the time spectra $\mathrm{TAC}_{i j}$ are "aligned" using energy-independent constant shift ${ }_{i j}$ values, which means that both the start and stop events from the detector-detector combination $i j$ are adjusted by the same shift ${ }_{i j}$ constant. As illustrated in Fig. 2b, this alignment does not introduce a systematic error and thus does not represent a correction, while it reduces the width of the distribution and thus the statistical error. These observations nicely confirm that the lifetime determination by means of centroid-shift measurements is independent of the shape of the PRF.

The major work of any fast-timing experiment is to calibrate the zero-time response of the set-up. Fig. 3 shows the (mean) PRD curve of the FATIMA plus the electronics set-up that was calibrated using $\gamma-\gamma$ cascades from a standard ${ }^{152} \mathrm{Eu} \gamma$-ray source and the in-beam reaction ${ }^{48} \mathrm{Ti}(\mathrm{n}, \gamma)^{49} \mathrm{Ti}[13]$. Some $82 \%$ of the data are nearly free of background due to the use of an additional EXILL (Ge) gate to select a triple $\gamma$-ray cascade. For the EXILL\&FATIMA experiments, a PRD uncertainty of 10 ps has been achieved which includes possible systematic errors [13].

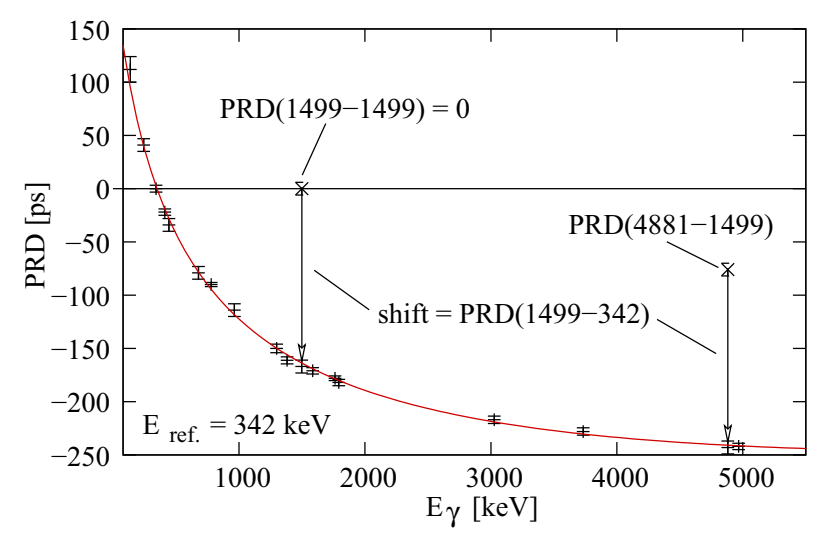

Figure 3. The (mean) PRD curve of the FATIMA plus the electronics set-up of the EXILL\&FATIMA spectrometers, adjusted for the refence energy of $342 \mathrm{keV}$. The PRD-calibration procedure is to adjust the two data points of prompt $\gamma-\gamma$ cascades by parallel shifts to fit a single smooth PRD curve. Referring to the decay transition of a cascade, the measured $\operatorname{PRD}\left(\Delta E_{\gamma}\right)$ is plotted at the energy of the feeding $\gamma$ ray, while the PRD at the decay energy vanishes according to Eqs. (9) and (10) and thus provides the second data point with same statistical uncertainty.

The major systematic error that can be induced in GCD analysis is due to the Compton background underneath the full-energy peak of interest which contributes to the FATIMA time spectra. Fig. 4 illustrates a test case on the lifetime determination of the $2_{1}^{+}$state in ${ }^{100} \mathrm{Zr}$. The results are from triple Ge-LaBr-LaBr (or Ge-LaBr-Ge) coin- cidences out of 8 TB of data digitally acquired triggerless from a 12 days measurement on cold-neutron-induced fission fragments of ${ }^{235} \mathrm{U}$. As shown in Fig. 4a, a relatively large Compton background is obtained due to high $\gamma$-ray multiplicity of over 100 nuclei produced in such experiments. However, the double-gated coincidence spectra are clean, meaning that no other $\gamma$ rays are observed in the vicinity of the peak at $352 \mathrm{keV}$, that could falsify the lifetime determination. The delayed and anti-delayed FATIMA time spectra of the 352-212-keV cascade shown in Fig. $4 \mathrm{~b}$ are nearly mirror symmetric which indicates the high quality of the FATIMA plus electronics set-up. By assuming the $\gamma$-Compton (full-energy peak vs. Compton) time response to be nearly prompt, the lifetime can be extraced directly from the slope of the time spectra [1].

The GCD method is sensitive to Compton background contributions to the time spectra. As illustrated in Fig. 4c, the 212-keV $\gamma$ vs. 352-keV Compton time response can be derived from a set of time spectra generated by setting gates in the Compton background around and above $352 \mathrm{keV}$. Taking into account the peak-to-background ratio $\Pi=2.6(2)$ of the $352-\mathrm{keV}$ peak in the FATIMA $\mathrm{LaBr}_{3}$ coincidence spectrum shown in Fig. $4 \mathrm{a}$, the net $\gamma-\gamma$ centroid difference $\Delta C_{\text {net }}$ is derived from the time shift [here +649 (20) ps] between the measured centroid difference and the $\gamma$-Compton time response $\Delta C_{\text {Compton }}$ at $352 \mathrm{keV}$ using $[3,13]$ :

$$
\Delta C_{\text {net }}=\Delta C+\frac{\Delta C-\Delta C_{\text {Compton }}}{\Pi} .
$$

By inserting the net centroid difference in Eq. (11) with $\operatorname{PRD}(352)=-61(10) \mathrm{ps}$, the mean lifetime of the first $2^{+}$state in ${ }^{100} \mathrm{Zr}$ of $\tau\left(2_{1}^{+}\right)=821(12)$ ps follows, which is in good agreement with the results obtained using the slope method and also confirms the experimental weighted average of $\tau=851$ (43) ps reported in Ref. [15]. Further tests and new measurements on short lifetimes down to the limit of about 10 ps also confirmed the method [13, 16$18]$.

\section{Conclusions}

At the PF1B collimated cold-neutron-beam line of the Institut Laue-Langevin, Ge-gated $\gamma-\gamma$ fast-timing measurements of excited states have been performed on around 80 nuclei from neutron-capture and neutron-induced fission experiments using $16 \mathrm{LaBr}_{3}(\mathrm{Ce})$ detectors. The complex data sets are analyzed using the newly developed GCD method which provides a simple algorithm and thus a rapid tool to generate the fast-timing-array time spectra. The mirror-symmetric GCD method is picosecond sensitive making it possible to derive a precise time correction related to the Compton background underneath the fullenergy peaks of the $\gamma-\gamma$ cascades. First high-precision results of lifetimes down to the limit of about 10 ps have been reported. The GCD method is a powerful tool which allows to test the set-up of a fast-timing array. Deviations from smooth $\gamma-\gamma$ time walk (the PRD curve) are quickly identified and indicate systematic shifts, e.g. induced by cross-talk events. 
a)

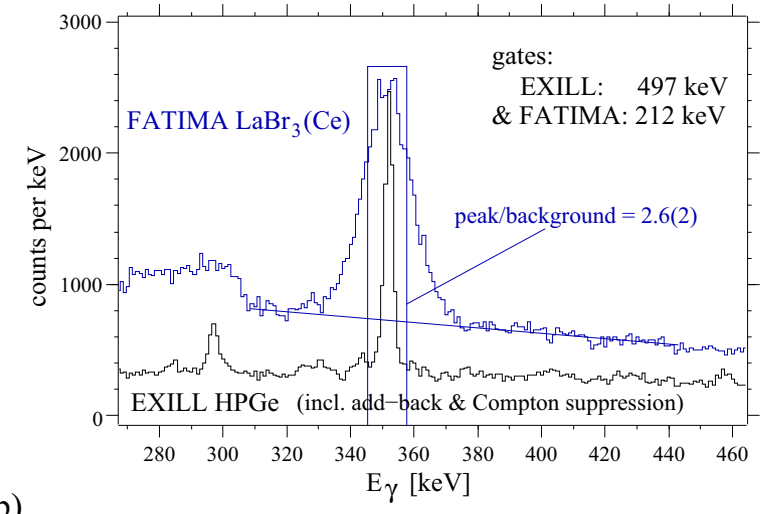

b)

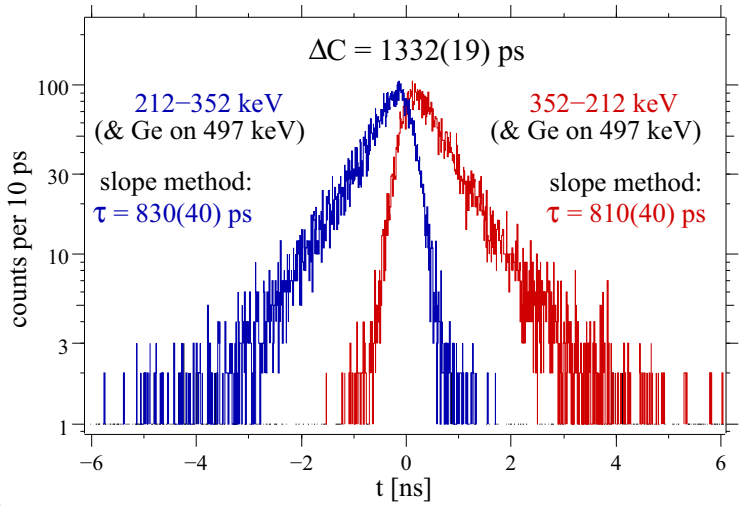

c)

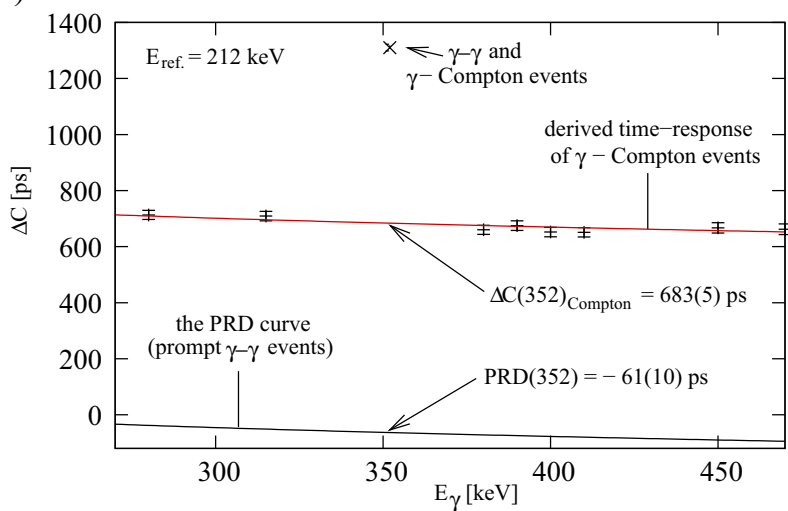

Figure 4. a: Comparison of the double-gated $(\mathrm{Ge}+\mathrm{LaBr})$ FATIMA and EXILL coincidence spectra, showing the cleanliness of the data in the region of the $352-\mathrm{keV}$ peak. b: The two EXILLgated FATIMA time spectra of the 352-212-keV $\gamma-\gamma$ cascade in ${ }^{100} \mathrm{Zr}$. According to the peak-to-background ratio, about $40 \%$ of the data are due to $\gamma$-Compton events. c: Illustration of the $\gamma$-Compton time-correction procedure by the centroid difference diagram for the reference energy of $212 \mathrm{keV}$ (common gate of all time spectra). The PRD curve is adjusted by a parallel shift to cross the energy axis at the reference energy.

\section{Acknowledgment}

This work was supported by NuPNET, by contracts 05P12PKNUF and 05P12RDNUP of the German BMBF, by contract DNC7RP01/4 of the British STFC and by the Spanish MINECO projects FPA2010-17142, CPAN (CSD 2007-00042) and PRI-PIMNUP-2011-1338 (via the ERA-NET NuPNET call for translational joint activities). The EXILL\&FATIMA campaign would not have been possible without the support of several services at the ILL and the LPSC. We are grateful to the EXOGAM collaboration for the loan of the detectors, to GANIL for assistance during installation and dismantling and to the FATIMA collaboration for the use of the $\mathrm{LaBr}_{3}(\mathrm{Ce})$ scintillator detectors.

\section{References}

[1] W. D. Hamilton, The electromagnetic interaction in nuclear spectroscopy, North-Holland New York 1975, 173

[2] H. Mach, R. L. Gill, M. Moszyński, Nuclear Instruments and Methods in Physics Research Section A 280, 49 (1989)

[3] J.-M. Régis, et al., Nuclear Instruments and Methods in Physics Research Section A 622, 83 (2010)

[4] A. Dewald, S. Harissopulos, P. von Brentano, Zeitschrift für Physik A 334, 163 (1989)

[5] A. Winther, K. Alder, Amsterdam, North-Holland 1975; New York, American Elsevier 1975

[6] Z. Bay, Physics Review 77, 419 (1950)

[7] H. Mach, et al., Nuclear Physics A 523, 197 (1991)

[8] J.-M. Régis, et al., Nuclear Instruments and Methods in Physics Research Section A 684, 36 (2012)

[9] J.-M. Régis, et al., Nuclear Instruments and Methods in Physics Research Section A 726, 191 (2013)

[10] G. de France, et al., EPJ Web of Conferences 66, 02010 (2014)

[11] H. Abele, et al., Nuclear Instruments and Methods in Physics Research Section A 562407 (2006)

[12] W. Urban, et al., Journal of Instrumentations 8, P03014 (2011)

[13] J.-M. Régis, et al., Nuclear Instruments and Methods in Physics Research Section A 763, 210 (2014)

[14] A. Kuronen, et al., Nuclear Physics A 549, 59 (1992)

[15] B. Singh, Nuclear Data Sheets 109, 297 (2008)

[16] J. Jolie, et al., these proceedings

[17] J. Jolie, et al., Journal of Physics: Conf. Ser. 533, 012026 (2014)

[18] S. Ilieva, et al., submitted to JPS Conf. Proc. (2014) 
